\title{
UNCERTAINTIES IN ESTIMATES OF THE RISKS OF LATE EFFECTS FROM SPACE RADIATION
}

\author{
F. A. Cucinotta ${ }^{1}$, W. Schimmerling ${ }^{2}$, J.W. Wilson ${ }^{3}$, L. E. Peterson ${ }^{4}$, P. Saganti ${ }^{1}$, and J. F. \\ Dicello
}

${ }^{1}$ NASA, Johnson Space Center, Houston TX $77058,{ }^{2}$ NASA, Headquarters, Washington D.C. 20546, ${ }^{3}$ NASA, Langley Research Center, Hampton VA 23681

${ }^{4}$ Department of Medicine, Baylor College of Medicine, Houston TX 77030

${ }^{5}$ Johns Hopkins Medical School, Baltimore MD 21287

Francis.A.Cucinotta1@jsc.nasa.gov 281-483-0968

The health risks faced by astronauts from space radiation include cancer, cataracts, hereditary effects, and non-cancer morbidity and mortality risks related to the diseases of the old age. Methods used to project risks in low-Earth orbit are of questionable merit for exploration missions because of the limited radiobiology data and knowledge of galactic cosmic ray (GCR) heavy ions, which causes estimates of the risk of late effects to be highly uncertain. Risk projections involve a product of many biological and physical factors, each of which has a differential range of uncertainty due to lack of data and knowledge. Within the linear-additivity model, we use Monte-Carlo sampling from subjective uncertainty distributions in each factor to obtain a Maximum Likelihood estimate of the overall uncertainty in risk projections. The resulting methodology is applied to several human space exploration mission scenarios including ISS, lunar station, deep space outpost, and Mar's missions of duration of 360, 660, and 1000 days. The major results are the quantification of the uncertainties in current risk estimates, the identification of factors that dominate risk projection uncertainties, and the development of a method to quantify candidate approaches to reduce uncertainties or mitigate risks. The large uncertainties in GCR risk projections lead to probability distributions of risk that mask any potential risk reduction using the "optimization" of shielding materials or configurations. In contrast, the design of shielding optimization approaches for solar particle events and trapped protons can be made at this time, and promising technologies can be shown to have merit using our approach. The methods used also make it possible to express risk management objectives in terms of quantitative objectives, i.e., the number of days in space without exceeding a given risk level within well defined confidence limits. 